\title{
A modified Li-He's variational principle for plasma
}

\author{
Ji-Huan $\mathrm{He}$ \\ National Engineering Laboratory for Modern Silk, \\ College of Textile and Clothing Engineering, Soochow University, Suzhou, China \\ and School of Mathematics and Information Science, \\ Henan Polytechnic University, Jiaozuo, China
}

\begin{abstract}
Purpose - It is extremely difficult to establish a variational principle for plasma. Kalaawy obtained a variational principle by using the semi-inverse method in 2016, and Li and He suggested a modification in 2017. This paper aims to search for a generalized variational formulation with a free parameter.

Design/methodology/approach - The semi-inverse method is used by suitable construction of a trial functional with some free parameters.

Findings - A modification of Li-He's variational principle with a free parameter is obtained.

Originality/value - This paper suggests a new approach to construction of a trial-functional with some free parameters.
\end{abstract}

Keywords Variational theory, Semi-inverse method, Burger equation, Plasma, Editorial

Paper type Research paper

\section{Introduction}

In 2016, Kalaawy obtained the following equation for plasma (El-Kalaawy, 2016):

$$
\frac{\partial u}{\partial t}+\frac{\partial}{\partial x}\left[\frac{2}{3} A u^{3 / 2}-C \frac{\partial u}{\partial x}\right]=0
$$

and derived a variational principle by using the semi-inverse method (He, 2004; He, 2017; Wu and He, 2018) through introducing two special functions.

In 2017, $\mathrm{Li}$ and $\mathrm{He}$ found another variational principle, which reads ( $\mathrm{Li}$ and $\mathrm{He}, 2017$ ):

$$
J_{L i-H e}(u, \Phi)=\iint\left\{\frac{1}{2} \frac{\partial \Phi}{\partial x} \frac{\partial \Phi}{\partial t}+\frac{2}{3} A u^{3 / 2} \frac{\partial \Phi}{\partial x}-\frac{1}{2} C\left(\frac{\partial \Phi}{\partial x}\right)^{2}-\frac{2}{5} A u^{5 / 2}\right\} d x d t
$$

where $\Phi$ satisfies the following relations:

$$
\begin{gathered}
\frac{\partial \Phi}{\partial x}=u \\
\frac{\partial \Phi}{\partial t}=-\frac{2}{3} A u^{3 / 2}+C \frac{\partial u}{\partial x}
\end{gathered}
$$

\section{General variational principle with a free parameter}

The semi-inverse method is widely used to search for variational principles directly from governing equations (El-Kalaawy, 2016; El-Kalaawy, 2017; Biswas et al., 2017). By the 
semi-inverse method (He, 2004; He, 2017; Wu and He, 2018), we can construct a trial31,5 functional in the form:

$$
J(u, \Phi)=\iint\left\{u \frac{\partial \Phi}{\partial t}+\left[\frac{2}{3} A u^{3 / 2}-C \frac{\partial u}{\partial x}\right] \frac{\partial \Phi}{\partial x}+F\right\} d x d t
$$

where $F$ is a known function of $\mathrm{u}$ and its derivative, however we cannot identify $F$, so equation (5) has to be modified as:

$J_{\text {New }}(u, \Phi)=\iint\left\{m u \frac{\partial \Phi}{\partial t}+n \frac{\partial \Phi}{\partial x} \frac{\partial \Phi}{\partial t}+a A u^{1 / 2}\left(\frac{\partial \Phi}{\partial x}\right)^{2}+b A u^{3 / 2} \frac{\partial \Phi}{\partial x}-C \frac{\partial u}{\partial x} \frac{\partial \Phi}{\partial x}+F\right\} d x d t$

where $m, n, a$ and $b$ are constants.

The stationary conditions of equation (6) are as follows:

$$
-m \frac{\partial u}{\partial t}-2 n \frac{\partial^{2} \Phi}{\partial x \partial t}-\frac{\partial}{\partial x}\left[2 a A u^{1 / 2} \frac{\partial \Phi}{\partial x}+b A u^{3 / 2}-C \frac{\partial u}{\partial x}\right]=0
$$

and

$$
m \frac{\partial \Phi}{\partial t}+\frac{1}{2} a A u^{-1 / 2}\left(\frac{\partial \Phi}{\partial x}\right)^{2}+\frac{3}{2} b A u^{1 / 2} \frac{\partial \Phi}{\partial x}+C \frac{\partial}{\partial x}\left(\frac{\partial \Phi}{\partial x}\right)+\frac{\delta F}{\delta u}=0
$$

In view of equations (3) and (4), we have:

$$
\begin{array}{r}
-(m+2 n) \frac{\partial u}{\partial t}-\frac{\partial}{\partial x}\left[(2 a+b) A u^{3 / 2}-C \frac{\partial u}{\partial x}\right]=0 \\
m C \frac{\partial u}{\partial x}+\left(\frac{1}{2} a+\frac{3}{2} b-\frac{2}{3} m\right) A u^{3 / 2}+C \frac{\partial u}{\partial x}+\frac{\delta F}{\delta u}=0
\end{array}
$$

Equation (9) should be equivalent to equation (1), this requires:

$$
\begin{aligned}
& 2 a+b=1 \\
& m+2 n=1
\end{aligned}
$$

To identify $\mathrm{F}$ in equation (10), we set:

$$
m=-1
$$

Equation (10) becomes:

$$
\left(\frac{1}{2} a+\frac{3}{2} b+\frac{2}{3}\right) A u^{3 / 2}+\frac{\delta F}{\delta u}=0
$$


From equation (14), F can be identified as:

$$
F=-\frac{2}{5}\left(\frac{1}{2} a+\frac{3}{2} b+\frac{2}{3}\right) A u^{5 / 2}=-\frac{2}{5}\left(\frac{13}{6}-\frac{5}{2} a\right) A u^{5 / 2}
$$

We, therefore, obtain the following variational principle:

$J_{\text {New }}(u, \Phi)=\iint\left\{\frac{\partial \Phi}{\partial x} \frac{\partial \Phi}{\partial t}-u \frac{\partial \Phi}{\partial t}+a A u^{1 / 2}\left(\frac{\partial \Phi}{\partial x}\right)^{2}+(1-2 a) A u^{3 / 2} \frac{\partial \Phi}{\partial x}-C \frac{\partial u}{\partial x} \frac{\partial \Phi}{\partial x}-\frac{2}{5}\left(\frac{13}{6}-\frac{5}{2} a\right) A u^{5 / 2}\right\} d x d t$

where $a$ is a free parameter.

\section{Conclusion}

In this paper, we suggest a general construction of a trial functional with some parameters. The semi-inverse method is a powerful tool to establish variational principles from the governing equations. The variational principle is a foundation of the variational iteration method (Anjum and He, 2019; He, 2006), which is now widely applied in fractional calculus (Baleanu et al., 2018; Dogan Durgun and Konuralp, 2018; Inc et al., 2018; Jafari et al., 2018; Wang et al., 2018).

\section{References}

Anjum, N. and He, J.H. (2019), "Laplace transform: Making the variational iteration method easier", Applied Mathematics Letters, Vol. 92, pp. 134-138.

Baleanu, D., Jassim, H.K. and Khan, H. (2018), "A modified fractional variational iteration method for solving nonlinear gas dynamic and coupled KdV equations involving local fractional operator", Thermal Science, Vol. 22 No. S1, pp. S165-S175.

Biswas, A., Zhou, Q., Moshokoa, S.P., Triki, H., Belic, M. and Alqahtani, R.T. (2017), "Resonant 1-soliton solution in anti-cubic nonlinear medium with perturbations", Optik, Vol. 145, pp. 14-17.

Dogan Durgun, D. and Konuralp, A. (2018), "Fractional variational iteration method for time-fractional nonlinear functional partial differential equation having proportional delays", Thermal Science, Vol. 22 No. S1, pp. S33-S46.

El-Kalaawy, O.H. (2016), "Variational principle, conservation laws and exact solutions for dust ion acoustic shock waves modeling modified Burger equation", Computers and Mathematics with Applications, Vol. 72, pp. 1013-1041.

El-Kalaawy, O.H. (2017), "New variational principle-exact solutions and conservation laws for modified ion-acoustic shock waves and double layers with electron degenerate in plasma", Physics of Plasmas, Vol. 24 No. 3, p. 32308.

He, J.H. (2004), "Variational principles for some nonlinear partial differential equations with variable coefficients", Chaos Solitons and Fractals, Vol. 19 No. 4, pp. 847-851.

He, J.H. (2006), "Some asymptotic methods for strongly nonlinear equations", International Journal of Modern Physics B, Vol. 20 No. 10, pp. 1141-1199.

He, J.H. (2017), "Hamilton's principle for dynamical elasticity", Applied Mathematics Letters, Vol. 72 No. 2017, pp. 65-69.

Inc, M., Khan, H., Baleanu, D. and Khan, A. (2018), "Modified variational iteration method for straight fins with temperature dependent thermal conductivity", Thermal Science, Vol. 22 No. S1, pp. S229-S236. 
HFF

31,5

Jafari, H., Jassim, H.K. and Vahidi, J. (2018), "Reduced differential transform and variational iteration methods for 3-D diffusion model in fractal heat transfer within local fractional operators", Thermal Science, Vol. 22 No. S1, pp. S301-S307.

Li, Y. and He, C.H. (2017), "A short remark on Kalaawy's variational principle for plasma”, International Journal of Numerical Methods for Heat and Fluid Flow, Vol. 27 No. 10, pp. 2203-2206.

Wang, Y., Zhang, Y.F. and Liu, Z.J. (2018), "An explanation of local fractional variational iteration method and its application to local fractional modified kortewed-de vries equation", Thermal Science, Vol. 22 No. 1, pp. 23-27.

Wu, Y. and He, J.H. (2018), “A remark on Samuelson's variational principle in economics”, Applied Mathematics Letters, Vol. 84, pp. 143-147.

\section{About the author}

Ji-Huan He is an expert on Nonlinear Science and Nanotechnology. He is the owner of some famous analytical methods, such as the semi-inverse method, the variational iteration method, the homotopy perturbation method, the exp-function method and He's frequency formulation. He has published more than 390 articles with an $h$-index of 66. Ji-Huan He can be contacted at: hejihuan@suda.edu.cn

For instructions on how to order reprints of this article, please visit our website: www.emeraldgrouppublishing.com/licensing/reprints.htm Or contact us for further details: permissions@emeraldinsight.com 